PANCREAS

\title{
Intravenous bolus somatostatin after diagnostic cholangiopancreatography reduces the incidence of pancreatitis associated with therapeutic endoscopic retrograde cholangiopancreatography procedures: a randomised controlled trial
}

\author{
R T-P Poon, C Yeung, C-L Liu, C-M Lam, W-K Yuen, C-M Lo, A Tang, S-T Fan
}

See end of article for authors' affiliations

Correspondence to:

Dr R T-P Poon, Department

of Surgery, Queen Mary

Hospital, 102 Pokfulam

Rd, Hong Kong, China;

poontp@hkucc.hku.hk

Accepted for publication 24 July 2003
Background: Previous studies suggested that somatostatin given before endoscopic retrograde cholangiopancreatography (ERCP) may reduce the incidence of post-ERCP pancreatitis. However, the routine use of somatostatin in all patients undergoing ERCP is not likely to be cost effective. This study evaluated whether intravenous bolus somatostatin given after diagnostic cholangiopancreatography could reduce the incidence of pancreatitis in a group of patients undergoing therapeutic ERCP procedures.

Methods: In a randomised, double blind, controlled trial, the effect of intravenous bolus somatostatin $250 \mu \mathrm{g}$ given immediately after diagnostic cholangiopancreatography was compared with that of placebo in patients who required endoscopic sphincterotomy or other therapeutic procedures. The primary end point was the incidence of post-ERCP clinical pancreatitis, and a secondary end point was the incidence of hyperamylasemia.

Results: A total of 270 patients were randomised. The somatostatin group $(n=135)$ and the placebo group $(n=135)$ were comparable in age, sex, indications for treatment, and types of procedure. The frequencies of clinical pancreatitis $(4.4 \%$ v $13.3 \% ; p=0.010)$ and hyperamylasemia $(26.0 \% v 38.5 \% ; p=0.036)$ were both significantly lower in the somatostatin group compared with the placebo group.

Conclusions: A single dose of intravenous bolus somatostatin, given immediately after diagnostic cholangiopancreatography, is effective in reducing the incidence of pancreatitis after therapeutic ERCP. This novel approach of administering prophylactic somatostatin may offer a cost effective prophylaxis for post-ERCP pancreatitis.
A cute pancreatitis is a common complication of endoscopic retrograde cholangiopancreatography (ERCP), with an incidence of $8-18 \%$ in prospective studies. ${ }^{1-5}$ Several agents have been tested for their efficacy in preventing post-ERCP pancreatitis. Somatostatin, ${ }^{67}$ octreotide, ${ }^{89}$ and gabexate ${ }^{10}$ are the three most widely investigated agents. A recent meta-analysis of randomised controlled trials showed that somatostatin and gabexate, but not octreotide, significantly reduced the incidence of post-ERCP pancreatitis. ${ }^{11}$ The study also found that somatostatin was more cost effective than gabexate from a pharmacoeconomic point of view as the number of patients that need to be treated to prevent one single episode of acute pancreatitis was 13 for somatostatin and 27 for gabexate. However, the study concluded that generalised treatment for all patients undergoing ERCP is unlikely to be cost effective and suggested that prophylactic therapy should be reserved for high risk patients who will benefit most from the treatment. ${ }^{11}$

Somatostatin can be administered as a continuous infusion or bolus intravenous injection before ERCP. A previous study from our institution demonstrated that somatostatin infusion started 30 minutes before ERCP and continued for 12 hours after the procedure reduced the incidence of post-ERCP pancreatitis. ${ }^{6}$ Most other studies on the effect of somatostatin on post-ERCP pancreatitis also employed intravenous infusion for 4-26 hours. ${ }^{12-14}$ However, continuous infusion increases the cost of the treatment and is impractical for ERCP as an outpatient procedure. ${ }^{11}{ }^{15}{ }^{16}$ In a recent study, a single bolus intravenous injection of somatostatin just before cannulation of the papilla significantly reduced the incidence of post-ERCP pancreatitis, especially in patients undergoing endoscopic sphincterotomy. ${ }^{7}$ This is a simpler and more cost effective way of administering somatostatin, and it may be useful in the outpatient setting. However, the generalised use of prophylactic somatostatin in a large number of patients to prevent a small number of cases of post-ERCP pancreatitis is still not justified in terms of cost effectiveness. Most cases of post-ERCP pancreatitis are mild and the main consequence is prolonged hospital stay. ${ }^{6}$

Ideally, somatostatin should be used only in patients with a high risk of post-ERCP pancreatitis to maximise the benefit-cost ratio. Previous studies have identified sphincter of Oddi dysfunction, non-dilated bile duct, difficult cannulation, repeated pancreatic duct injection, pancreatic duct acinarisation, endoscopic sphincterotomy, and therapeutic procedures as risk factors for post-ERCP pancreatitis. ${ }^{2}{ }^{17-21}$ Although patient factors such as sphincter of Oddi dysfunction and non-dilated bile duct may be known before ERCP, technical factors are largely unpredictable before the procedure. Even for patients who are likely to require a therapeutic procedure, the decision of whether to proceed to

Abbreviations: $C B D$, common bile duct; $E R C P$, endoscopic retrograde cholangiopancreatography 
sphincterotomy or other therapeutic procedures is made only after successful cannulation and assessment of findings at diagnostic cholangiopancreatography. Hence except for a few cases with well defined risk factors, selective use of prophylactic somatostatin in high risk patients before cannulation is not practical in most circumstances.

The benefit of prophylactic somatostatin would be more pronounced if a bolus injection during the procedure immediately after diagnostic cholangiopancreatography could reduce the risk of pancreatitis associated with sphincterotomy or other therapeutic procedures, as dictated by the findings of cholangiopancreatography. To test this hypothesis, we conducted a randomised controlled trial to evaluate the efficacy of bolus intravenous somatostatin given immediately after diagnostic cholangiopancreatography in reducing the incidence of pancreatitis in patients who required sphincterotomy or other therapeutic procedures.

\section{PATIENTS AND METHODS \\ Eligibility}

A randomised, double blind, controlled trial was conducted in the Endoscopy Unit of the Department of Surgery, University of Hong Kong, Queen Mary Hospital, Hong Kong. Eligible patients were those undergoing a first therapeutic ERCP, with sphincterotomy or other therapeutic procedures deemed necessary after a diagnostic cholangiopancreatography. Exclusion criteria included: (1) age $<18$ years; (2) pregnancy; (3) previous sphincterotomy or therapeutic ERCP; (4) acute myocardial infarction within three months; (5) acute pancreatitis or hyperamylasemia at the baseline blood test; (6) chronic pancreatitis; and (7) history of allergic reaction to somatostatin. Informed consent was obtained from all patients who participated in the study, which was approved by the local ethics committee.

\section{Study design}

Before undergoing ERCP, all patients were fasted for six hours. One dose of intravenous prophylactic antibiotic (amoxicillin-clavulanic acid $1.2 \mathrm{~g}$ ) was given before the procedure and intravenous sedation was provided using meperidine and midazolam. All procedures were performed by fully trained endoscopists experienced in therapeutic ERCP.

All patients underwent a diagnostic cholangiopancreatography first. For patients with suspected biliary disease, selective cannulation of the common bile duct (CBD) was attempted without cannulation or injection of the pancreatic duct whenever possible. Pancreatic duct cannulation or contrast injection was performed only if indicated. Patients who required sphincterotomy or other therapeutic procedures based on the findings of cholangiopancreatography were randomised into one of two groups: (1) the somatostatin group received an intravenous bolus injection of $250 \mu \mathrm{g}$ somatostatin (Somatostatin-UCB; UCB Pharma, Brussels, Belgium) immediately after the diagnostic cholangiopancreatography but before sphincterotomy or other therapeutic procedures in the same session; (2) the placebo group received a bolus of the same volume of intravenous normal saline immediately after the diagnostic cholangiopancreatography. Therapeutic procedures in this study included sphincterotomy, pre-cut papillotomy, stenting, stone extraction, or balloon dilatation of the sphincter of Oddi. Only patients requiring a therapeutic procedure were included in the study.

Randomisation was carried out only after the diagnostic cholangiopancreatography was obtained and the need for therapeutic interventions was decided. Randomisation was performed by a nurse, who was not involved in the procedure or any part of patient care, by opening numbered sealed envelopes containing computer generated random numbers assigning patients to the somatostatin or placebo group without stratification. The sealed envelopes and drugs were kept in the endoscopy suite. Somatostatin or placebo injection was given by the same nurse who performed the randomisation. The endoscopist, the nurse assisting the procedure, and the patient were all blind to the treatment allocation. The therapeutic procedure was then performed after the injection was given. Sphincterotomy was performed using pure cut current with an output power of $30 \mathrm{~W}$.

The endoscopists completed a data collection sheet immediately after finishing the therapeutic procedure. CBD cannulation was rated by the endoscopists as "easy" or "difficult" (requiring three or more attempts to achieve deep cannulation of the CBD). Details of the endoscopic findings and procedures were recorded.

After ERCP, all patients were fasted for at least six hours and stayed overnight for 24 hours, as is the policy of our institution. Serum amylase was measured before ERCP (baseline) and at six and 24 hours afterwards. Clinical parameters including abdominal pain, tenderness, and requirement for analgesia (intramuscular meperidine) were documented during the 24 hours after the procedure by the physicians in the ward, who were not aware of whether the patient had received somatostatin or placebo during ERCP. All other ERCP related complications were noted. For patients who developed post-ERCP pancreatitis, serum amylase was monitored daily until it returned to normal. Patients continued to fast in the hospital until amylase levels returned to normal and pain subsided.

\section{Study outcomes}

The primary outcome of the study was the incidence of postERCP clinical pancreatitis, which is defined as new or worsened abdominal pain and tenderness persisting for more than 24 hours after endoscopy with a raised serum amylase level more than three times the upper normal limit ( $110 \mathrm{IU} / \mathrm{l}$ ) at 24 hours. ${ }^{6}{ }^{17}$ Severity of pancreatitis was classified as mild when the length of stay was $\leqslant 3$ nights, moderate when hospital stay was 4-10 nights, and severe if more than 10 days of hospitalisation, intensive care unit admission, or surgery was needed for pancreatitis. ${ }^{17}$ For patients who required further hospital stay for other reasons after resolution of post-ERCP pancreatitis, the number of days from the date of ERCP until the date of resolution of abdominal pain and normalisation of serum amylase levels was taken as hospital stay due to pancreatitis. A secondary outcome was the incidence of hyperamylasemia, which was defined as an elevation in serum amylase levels to greater than three times the upper normal limit at either six or 24 hours after ERCP.

\section{Statistical analysis}

Based on previous studies, ${ }^{67}$ it was estimated that the incidence of post-ERCP pancreatitis after endoscopic sphincterotomy or therapeutic ERCP procedures in the placebo group would be approximately $15 \%$. Sample size calculations showed that 130 patients in each group were needed for an $\alpha$ error of 0.05 and a $\beta$ error of 0.20 , based on the assumption that somatostatin could reduce the incidence of pancreatitis from $15 \%$ to $5 \%$.

The $\chi^{2}$ test (or Fisher's exact test where appropriate) was used for comparison of categorical data. All continuous data were expressed as median with interquartile range and compared using the Mann-Whitney U test. A p value $<0.05$ was considered statistically significant. All analyses were performed using statistical software (SPSS 9.0; SPSS, Inc, Chicago, Illinois, USA). 


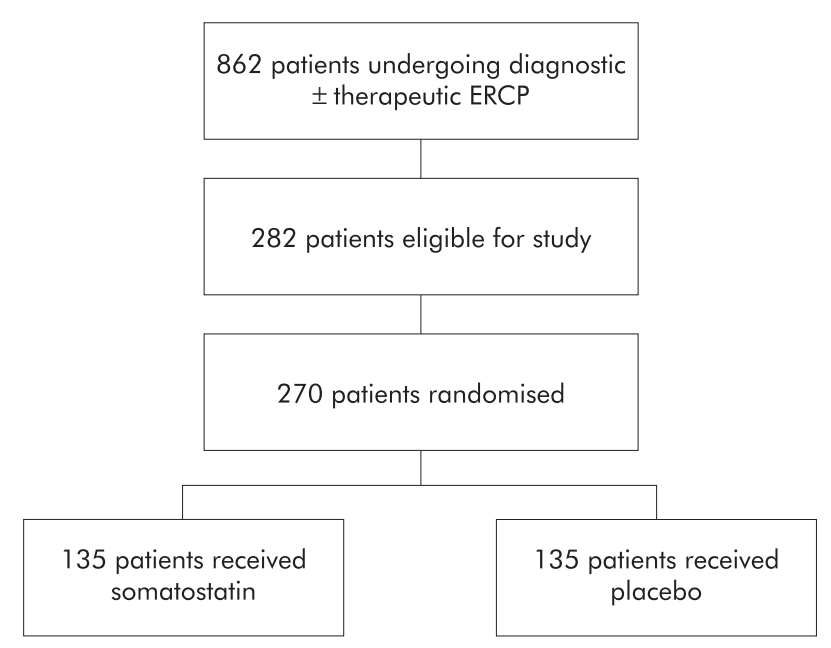

Figure 1 Trial profile. ERCP, endoscopic retrograde cholangiopancreatography.

\section{RESULTS}

Over a period of 24 months from July 2000 to June 2002, 270 patients were randomised, with 135 patients in each of the treatment arms (fig 1). Data were complete except in three patients who had either the six hour $(n=2)$ or 24 hour $(\mathrm{n}=1)$ post-ERCP serum amylase level not checked. These three patients were included in the analysis.

\section{Clinical and endoscopic data}

The two groups were comparable in terms of age, sex, incidence of previous pancreatitis, and indications for therapeutic ERCP (table 1). The most common indication for therapeutic ERCP was CBD stone. Therapeutic manoeuvres and endoscopic observations are shown in table 2 . Overall, 190 of 270 patients (70.4\%) had endoscopic sphincterotomies, all of which were biliary sphincterotomies. There were no significant differences between the two groups in the proportion of patients requiring endoscopic sphincterotomy or other therapeutic procedures. The two groups also had similar proportions of patients with non-dilated bile duct, difficult CBD cannulation, repeated pancreatic duct injection, and pancreatic acinarisation, which were endoscopic observations that might influence the incidence of pancreatitis.

\section{Post-ERCP serum amylase levels}

There was no significant difference in pre-ERCP serum amylase levels between the two groups (median 69 (53-86) $v 72(56-88) \mathrm{IU} / \mathrm{l} ; \mathrm{p}=0.433)$. However, both the six hour post-ERCP serum amylase level (median 135 (74-358) v 210 (87-679) IU/l; $p=0.028$ ) and the 24 hour post-ERCP serum

\begin{tabular}{|c|c|c|}
\hline & Somatostatin group $(n=135)$ & Placebo group $(n=135$ ) \\
\hline $\operatorname{Sex}(M / F)$ & $65 / 70$ & $72 / 63$ \\
\hline Age $(y)$ & $69(56-76)$ & $67(52-75)$ \\
\hline Previous pancreatitis & $8(5.9 \%)$ & $6(4.4 \%)$ \\
\hline \multicolumn{3}{|l|}{ Diagnosis } \\
\hline CBD stone & $94(69.6 \%)$ & $94(69.6 \%)$ \\
\hline Intrahepatic ductal stone & $12(8.9 \%)$ & $10(7.4 \%)$ \\
\hline Carcinoma of pancreas & $10(7.4 \%)$ & $12(8.9 \%)$ \\
\hline Carcinoma of CBD & $6(4.4 \%)$ & $6(4.4 \%)$ \\
\hline Carcinoma of ampulla & $4(3.0 \%)$ & $3(2.2 \%)$ \\
\hline Klatskin tumour & $5(3.7 \%)$ & $6(4.4 \%)$ \\
\hline Hepatocellular carcinoma & $2(1.5 \%)$ & $2(1.5 \%)$ \\
\hline Pancreatic cystoadenoma & $0(0 \%)$ & $1(0.7 \%)$ \\
\hline Choledochal cyst & $1(0.7 \%)$ & $0(0 \%)$ \\
\hline Post-cholecystectomy bile leakage & $1(0.7 \%)$ & $1(0.7 \%)$ \\
\hline \multicolumn{3}{|c|}{$\begin{array}{l}\text { Age is expressed as median (interquartile range). All other values are number (\%). } \\
C B D \text {, common bile duct. }\end{array}$} \\
\hline
\end{tabular}

Table 2 Therapeutic procedures and other endoscopic findings

\begin{tabular}{|c|c|c|c|}
\hline & $\begin{array}{l}\text { Somatostatin group } \\
(n=135)\end{array}$ & $\begin{array}{l}\text { Placebo group } \\
(n=135)\end{array}$ & p Value \\
\hline \multicolumn{4}{|l|}{ Therapeutic procedure* } \\
\hline Biliary sphincterotomy & $98(72.6 \%)$ & $92(68.1 \%)$ & 0.424 \\
\hline Precut & $6(4.4 \%)$ & $5(3.7 \%)$ & 0.758 \\
\hline Biliary stenting & $47(34.8 \%)$ & $54(40.0 \%)$ & 0.379 \\
\hline CBD stone extraction & $62(45.9 \%)$ & $51(37.8 \%)$ & 0.175 \\
\hline \multicolumn{4}{|l|}{ Balloon dilation of sphincter of } \\
\hline Difficult cannulation $†$ & $58(43.0 \%)$ & $56(41.5 \%)$ & 0.805 \\
\hline Non-dilated CBD & $44(32.6 \%)$ & $46(34.1 \%)$ & 0.796 \\
\hline Pancreatic duct cannulation & $38(28.1 \%)$ & $42(31.1 \%)$ & 0.594 \\
\hline \multicolumn{4}{|l|}{ Repeated ( $\geqslant 3$ times) pancreatic } \\
\hline Pancreatic acinarisation & $3(2.2 \%)$ & $4(3.0 \%)$ & 1.000 \\
\hline $\begin{array}{l}\text { Values are number (\%). } \\
\text { CBD, common bile duct. } \\
\text { *All patients had at least one the } \\
\text { †Required more than three attem }\end{array}$ & $\begin{array}{l}\text { eutic procedure; sc } \\
\text { at cannulation. }\end{array}$ & its had more th & edure. \\
\hline
\end{tabular}


Table 3 Incidence of post-endoscopic retrograde cholangiopancreatography hyperamylasemia, abdominal pain, and clinical pancreatitis

\begin{tabular}{llll}
\hline & $\begin{array}{l}\text { Somatostatin group } \\
(\mathbf{n}=135)\end{array}$ & $\begin{array}{l}\text { Placebo group } \\
(\mathbf{n}=135)\end{array}$ & $\mathbf{p}$ Value \\
\hline Hyperamylasemia & $35(26.0 \%)$ & $52(38.5 \%)$ & 0.036 \\
Abdominal pain & $16(12.0 \%)$ & $27(20.0 \%)$ & 0.067 \\
Clinical pancreatitis & $6(4.4 \%)$ & & \\
All & 3 & $18(13.3 \%)$ & 0.010 \\
Mild & 3 & 9 & \\
Moderate & 0 & 0 & \\
Severe & & & \\
\hline
\end{tabular}

amylase level (median 128 (2-237) $v$ l82 (6-605) IU/l; $\mathrm{p}=0.007$ ) were significantly lower in the somatostatin group than in the placebo group. Hyperamylasemia was observed less frequently in the somatostatin group than in the placebo group $(\mathrm{p}=0.036$, table 3$)$.

\section{Post-ERCP clinical pancreatitis}

Sixteen patients $(12.0 \%)$ in the somatostatin group and 27 patients $(20.0 \%)$ in the placebo group developed abdominal pain (or worsening of abdominal pain) after ERCP $(\mathrm{p}=0.067$, table 3$)$. The incidence of clinical pancreatitis was significantly lower in the somatostatin group compared with the placebo group $(4.4 \% \vee 13.3 \% ; \mathrm{p}=0.010)$. Based on our results, 11 patients need to be treated with somatostatin to prevent one case of post-ERCP pancreatitis. There were no significant differences in the frequencies of pancreatitis between patients who had undergone sphincterotomy and those who had a therapeutic procedure without sphincterotomy within the somatostatin group $(4.1 \% \vee 5.4 \% ; \mathrm{p}=0.666)$ or the placebo group $(13.0 \% v 14.0 \% ; \mathrm{p}=0.885)$.

Three patients in the somatostatin group and nine patients in the placebo group had moderate pancreatitis; the others had mild pancreatitis. None of these patients required intensive care unit admission or surgical intervention. The median duration of post-ERCP hospital stay in the somatostatin and placebo groups was $2(1-3)$ and 3 (1-4) days, respectively $(\mathrm{p}=0.048)$. More specifically, the total extra hospital stay due to post-ERCP pancreatitis in the somatostatin and placebo groups was 48 and 94 days, respectively. Use of somatostatin resulted in a reduction of 0.34 days of hospital stay per patient treated.

In addition to post-ERCP pancreatitis, post-sphincterotomy bleeding requiring endoscopic haemostasis occurred in two patients in each group, respectively. One patient in the somatostatin group had post-sphincterotomy duodenal perforation that was managed conservatively, and one patient in the placebo group developed acute cholangitis after ERCP. There were no deaths related to ERCP. No adverse effect attributable to somatostatin was observed.

\section{DISCUSSION}

Despite the technical improvements in recent years and the increased expertise of operators, the incidence of pancreatitis after ERCP and endoscopic sphincterotomy has not decreased.22 The search for an effective prophylactic therapy for post-ERCP pancreatitis is important. Studies on steroids, ${ }^{3}{ }^{4}$ allopurinol, ${ }^{4}$ and nifedipine ${ }^{5}$ have yielded disappointing results. Currently, somatostatin and gabexate appear to be agents that may be effective in preventing post-ERCP pancreatitis. ${ }^{671011}$ Both somatostatin and octreotide are potent inhibitors of exocrine pancreatic secretion. However, unlike somatostatin, octreotide has not been found to reduce post-ERCP pancreatitis. ${ }^{89}$ The exact reason for the different effects of somatostatin and octreotide remains unclear. ${ }^{23}$ One possible explanation might be that octreotide increases the basal pressure of the sphincter of $\mathrm{Oddi}^{24}$ whereas somatostatin inhibits sphincter contraction. ${ }^{25}$ Octreotide is usually given at doses of $0.1 \mathrm{mg}$ or $0.2 \mathrm{mg}$ by subcutaneous injection before ERCP, and it has been suggested that the dosage used might not have an effect on pancreatic enzyme secretion. ${ }^{9}{ }^{11} \mathrm{~A}$ recent randomised study suggested that interleukin 10 , which is a potent anti-inflammatory cytokine, may reduce the incidence of pancreatitis after therapeutic ERCP. ${ }^{26}$ However, another trial found that interleukin 10 was not effective. ${ }^{27}$

The ideal pharmacological prevention of post-ERCP pancreatitis should meet the following three criteria: (1) effective in patients who really risk developing post-ERCP pancreatitis; (2) not require prolonged administration in the postprocedure period; and (3) be as economical as possible to make it cost effective. Of the currently available drugs that may be useful, somatostatin is the least expensive. Although early randomised trials failed to demonstrate a significant benefit of somatostatin, ${ }^{12}{ }^{14}$ the sample size in these trials with fewer than 30 patients in each arm was not adequate. Our previous study involving 220 patients showed that continuous infusion of somatostatin for 12 hours after ERCP was effective in reducing post-ERCP pancreatitis. ${ }^{6}$ Another group, in a study of 160 patients, demonstrated that intravenous bolus somatostatin before ERCP reduced post-ERCP pancreatitis, especially after sphincterotomy. ${ }^{7}$ Intravenous bolus injection is a less costly way of administering somatostatin, but the use of somatostatin for all patients undergoing ERCP may not be cost effective, even with bolus injection.?

In the current trial, we demonstrated that an intravenous bolus injection of somatostatin after diagnostic cholangiopancreatography reduced the incidence of post-ERCP hyperamylasemia and pancreatitis in selected patients undergoing sphincterotomy or other therapeutic procedures. There was also a trend towards reduced frequency of abdominal pain in the somatostatin group, although this was not significantly different from the placebo group. The reduced incidence of post-ERCP abdominal pain was mainly due to the decrease in the frequency of pancreatitis, as the number of patients with abdominal pain not related to pancreatitis was similar between the two groups ( 10 and nine patients, respectively). In all of the previous trials on pharmacological prevention of post-ERCP pancreatitis, the prophylactic agent was given before ERCP, which did not allow selective use of the agent in high risk patients, except in those with known risk factors. This is a major drawback that has limited the clinical application of any drug for prophylaxis of post-ERCP pancreatitis because the main risk factors for post-ERCP pancreatitis were related to the procedure, and hence the risk of pancreatitis could only be predicted after diagnostic cholangiopancreatography. Our novel approach of selective use of somatostatin for high risk patients after diagnostic 
cholangiopancreatography allows the most cost effective use of the drug to prevent post-ERCP pancreatitis.

In accordance with the findings of other studies, ${ }^{6}{ }^{79}$ most cases of post-ERCP pancreatitis in this study were mild. Mortality from post-ERCP pancreatitis is rare. The main consequence of this complication is prolonged hospital stay. Hence it is important to consider the reduction in hospital stay for pancreatitis using prophylactic somatostatin versus the cost of the therapy. This has not been evaluated in previous studies. In this study, somatostatin resulted in an average reduction of 0.34 days of hospital stay per patient treated. In our institution, the cost of one dose of somatostatin was US\$15.00, whereas the average cost of 0.34 days of extra hospital stay is US\$130.00 per patient. Hence the use of prophylactic somatostatin in the setting of this trial was cost effective. The cost-benefit ratio will be further improved if the loss of working days of patients suffering from post-ERCP pancreatitis is taken into account. Our approach of administering somatostatin has a major advantage of being applicable to outpatient or day case ERCP practice, which is the current trend of practice even for therapeutic ERCP procedures. ${ }^{15}$

In this study, we chose patients undergoing endoscopic sphincterotomy or other therapeutic procedures as study subjects. Previous studies have shown that such patients are at a higher risk of post-PRCP pancreatitis than those undergoing diagnostic ERCP alone, presumably because of the thermal or mechanical injury to papilla and pancreatic orifice. ${ }^{217} 1820$ Although one recent study found that biliary sphincterotomy was not an independent risk factor for postERCP pancreatitis, ${ }^{28}$ other studies have demonstrated a higher frequency of post-ERCP pancreatitis associated with biliary sphincterotomy. ${ }^{2620}$ The relatively high incidence of post-ERCP pancreatitis in our placebo group (13.3\%) probably reflected prospective data collection. Other recent prospective studies have reported even higher frequencies of post-ERCP pancreatitis $(18-24 \%)$ in the placebo group after sphincterotomy or other therapeutic procedures. ${ }^{726}$ In addition to sphincterotomy and therapeutic procedures, patient factors such as the size of the CBD and other technical factors such as difficult CBD cannulation, could also affect the risk of post-ERCP pancreatitis. However, given that the frequencies of such endoscopic observations were similar between the two groups, it is unlikely that there was significant bias related to these factors.

It is possible that some degree of pancreatic injury might have already been incurred while obtaining a cholangiopancreatography before sphincterotomy or other therapeutic procedures. A delay of a few hours typically exists between the pancreatic injury and the peak of hyperamylasemia or onset of symptoms in post-ERCP pancreatitis. ${ }^{29}$ This provides a "therapeutic window" for drugs to work after initiation of the pancreatic injury. Coupled with the rapid onset of action of bolus intravenous somatostatin, this may explain the efficacy of the drug in reducing the incidence of pancreatitis even when it was given after instead of before diagnostic cholangiopancreatography. In addition to its effect on pancreatic enzyme secretion, recent evidence suggested that somatostatin could reduce the inflammatory reaction of acute pancreatitis through modulation of the cytokine cascade and induction of apoptosis in pancreatic acinar cells. ${ }^{30} 31$ This anti-inflammatory effect of somatostatin may be another mechanism for its efficacy in preventing postERCP pancreatitis when given as a bolus injection after diagnostic ERCP. A recent meta-analysis of clinical trials suggested that somatostatin could reduce the mortality of severe acute pancreatitis. ${ }^{32}$ The use of somatostatin at the initial onset of pancreatic inflammation appears to be a rational approach to prevent post-ERCP pancreatitis.
In this trial, we confined the study to patients undergoing therapeutic procedures, and most of the therapeutic procedures were performed for biliary diseases. The results cannot be generalised to other situations that predispose to postERCP pancreatitis. Repeated pancreatic duct injection, pancreatic acinarisation, and pancreatic therapeutic procedures were also risk factors for post-ERCP pancreatitis that were unpredictable before ERCP. ${ }^{21}{ }^{28}$ The use of somatostatin in such circumstances warrants further investigation.

In conclusion, this study shows that a single dose of intravenous bolus somatostatin given immediately after diagnostic cholangiopancreatography can decrease the incidence of pancreatitis after sphincterotomy or other therapeutic ERCP procedures. Our study provides insight into a new direction for future research on a more cost effective approach of using pharmacological agents to prevent postERCP pancreatitis. The use of the cheapest drug available, administered in the least costly way to a selected high risk group of patients, as demonstrated in this study, may offer the real possibility of cost effective prophylaxis of post-ERCP pancreatitis.

\section{Authors' affiliations}

R T-P Poon, C Yeung, C-L Liu, C-M Lam, W-K Yuen, C-M Lo, A Tang, S-T Fan, Surgical Endoscopy Unit, Department of Surgery, University of Hong Kong Medical Centre, Queen Mary Hospital, Hong Kong, China

\section{REFERENCES}

1 Sherman S, Hawes RH, Rathgaber SW, et al. Post-ERCP pancreatitis: randomized, prospective study comparing a low- and high-osmolality contrast agent Gastrointest Endosc 1994:40:422-7.

2 Gottlieb K, Sherman S, Pezzi J, et al. Early recognition of post-ERCP pancreatitis by clinical assessment and serum pancreatic enzymes. Am J Gastroenterol 1996;91:1553-7.

3 Dumot JA, Conwell DL, O'Connor JB, et al. Pretreatment with methylprednisolone to prevent ERCP-induced pancreatitis: a randomized, multicenter, placebo-controlled clinical trial. Am J Gastroenterol 1998;93:61-5

4 Budzynska A, Marek T, Nowak A, et al. A prospective, randomized, placebocontrolled trial of prednisone and allopurinol in the prevention of ERCPinduced pancreatitis. Endoscopy 2001;33:766-72.

5 Prat F, Amaris J, Ducot B, et al. Nifedipine for prevention of post-ERCP pancreatitis: a prospective, double-blind randomized study. Gastrointest Endosc 2002:56:202-8.

6 Poon RT, Yeung C, Lo CM, et al. Prophylactic effect of somatostatin on postERCP pancreatitis: a randomized controlled trial. Gastrointest Endosc 1999;49:593-8.

7 Bordas JM, Toledo-Pimentel V, Llach J, et al. Effects of bolus somatostatin in preventing pancreatitis after endoscopic pancreatography: results of a randomized study. Gastrointest Endosc 1998;47:230-4.

8 Binmoeller KF, Harris AG, Dumas R, et al. Does the somatostatin analogue octreotide protect against ERCP induced pancreatitis? Gut 1992;33:1129-33.

9 Testoni PA, Bagnolo F, Andriulli A, et al. Octreotide 24-h prophylaxis in patients at high risk for post-ERCP pancreatitis: results of a multicenter, randomized, controlled trial. Aliment Pharmacol Ther 2001;15:965-72.

10 Cavallini G, Tittobello A, Frulloni L, et al. Gabexate for the prevention of pancreatic damage related to endoscopic retrograde cholangiopancreatography. Gabexate in digestive endoscopy-Italian Group. N Engl J Med 1996;335:919-23.

11 Andriulli A, Leandro G, Niro G, et al. Pharmacologic treatment can prevent pancreatic injury after ERCP: a meta-analysis. Gastrointest Endosc 2000:51:1-7.

12 Testoni PA, Masci E, Bagnolo F, et al. Endoscopic papillo-sphincterotomy: prevention of pancreatic reaction by somatostatin. Ital J Gastroenterol 1988;20:70-3

13 Guelrud M, Mendoza S, Viera L, et al. Somatostatin prevents acute pancreatitis after pancreatic duct sphincter hydrostatic balloon dilation in patients with idiopathic recurrent pancreatitis. Gastrointest Endosc 1991;37:44-7

14 Persson B, Slezak P, Efendic S, et al. Can somatostatin prevent injection pancreatitis after ERCP? Hepatogastroenterology 1992:39:259-61.

15 Freeman ML, Nelson DB, Sherman S, et al. Same day discharge after endoscopic biliary sphincterotomy: observations from a prospective multicenter complication study. The Multicenter Endoscopic Sphincterotomy (MESH) Study Group. Gastrointest Endosc 1999;49:580-6.

16 Ho KY, Montes H, Sossenheimer MJ, et al. Features that may predict hospital admission following outpatient therapeutic ERCP. Gastrointest Endosc 1999;49:587-92.

17 Cotton PB, Lehman G, Vennes J, et al. Endoscopic sphincterotomy complications and their management: an attempt at consensus. Gastrointest Endosc 1991;37:383-93. 
18 Sherman S, Lehman GA. ERCP- and endoscopic sphincterotomy-induced pancreatitis. Pancreas 1991;6:350-67.

19 Sherman S, Ruffolo TA, Hawes RH, et al. Complications of endoscopic sphincterotomy: a prospective series with emphasis on the increased risk associated with sphincter of Oddi dysfunction and nondilated bile ducts. Gastroenterology 1991;101:1068-75.

20 Johnson GK, Geenen JE, Bedford RA, et al. A comparison of nonionic versus ionic contrast media: results of a prospective multicenter study. Midwest Pancreaticobiliary Study Group. Gastrointest Endosc 1995;42:312-16.

21 Vandervoort J, Soetikno RM, Tham TC, et al. Risk factors for complications after performance of ERCP. Gastrointest Endosc 2002;56:652-6.

22 Freeman ML, Nelson DB, Sherman S, et al. Complications of endoscopic biliary sphincterotomy. N Engl J Med 1996;335:909-18.

23 Poon RT, Fan ST. Antisecretory agents for prevention of post-ERCP pancreatitis: rationale for use and clinical results. JOP 2003;4:33-40.

24 Binmoeller KF, Dumas R, Harris AG, et al. Effect of somatostatin analog octreotide on human sphincter of Oddi. Dig Dis Sci 1992;37:773-7.

25 Ahrendt SA, McGuire GE, Lillemore KD, et al. Somatostatin inhibits sphincter of Oddi motility. Gastroenterology 1990;98:A242.
26 Devière J, Le Moine $O$, Van Laethem JL, et al. Interleukin-10 reduces the incidence of pancreatitis after therapeutic endoscopic retrograde cholangiopancreatography. Gastroenterology 2001;120:498-505.

27 Dumot JA, Conwell DL, Zuccaro G Jr, et al. A randomized, double blind study of interleukin 10 for the prevention of ERCP-induced pancreatitis. Am J Gastroenterol 2001;96:2098-102.

28 Freeman ML, DiSario JA, Nelson DB, et al. Risk factors for post-ERCP pancreatitis: a prospective, multicenter study. Gastrointest Endosc 2001;54:425-34

29 Messman H, Vogt W, Holstege A, et al. Post-ERCP pancreatitis as a model for cytokine induced acute phase response in acute pancreatitis. Gut 1997;40:80-5

30 Karalis K, Mastorakos G, Chrousos GP, et al. Somatostatin analogues suppress the inflammatory reaction in vivo. J Clin Invest 1994;93:2000-6.

31 Yuan Y, Gong Z, Lou K, et al. Effects and mechanisms of somatostatin analogs on apoptosis of pancreatic acinar cells in acute pancreatitis in mice. $J$ Gastroenterol Hepatol 2001;16:683-8.

32 Andriulli A, Leandro G, Clemente R, et al. Meta-analysis of somatostatin, octreotide and gabexate mesilate in the therapy of acute pancreatitis. Aliment Pharmacol Ther 1998;12:237-45.

\section{GI SNAPSHOT}

Answer

From question on page 1727

This contrast enhanced computed tomography image shows a large mass within the right lobe of the liver, measuring $17 \times 13 \mathrm{~cm}$. Because of the peripheral enhancement of the mass, and the central filling seen on delayed images, this finding was most consistent with giant haemangioma. This presumptive diagnosis was confirmed by a nuclear medicine red blood cell scan. After other infectious causes were ruled out, the patient's fever was felt to be due to this mass. The patient underwent a right hepatic lobectomy, which he tolerated well. His fevers resolved from postoperative day 3 onwards. He was discharged on postoperative day 8 and remained asymptomatic at his follow up appointments in clinic for two months. The final pathological diagnosis and cause of this patient's fever of unknown origin was a giant haemangioma with foci of thrombosis and infarction. 